\title{
LA STRUCTURE TEMPORELLE ET SPATIALE DANS LE ROMAN DE DEZSÖ MALONYAY INTITULE " AZ UTOLSÓ » (LE DERNIER)
}

\author{
LÁSZLÓ GERGYE \\ Université Eötvös Loránd de Budapest \\ dr.gergye@freemail.hu
}

\begin{abstract}
Résumé
L'article est consacré à un roman artistique peu connu de Dezső Malonyay intitulé «Az utolsó » (Le dernier), spécialement sous l'aspect de la gestion du temps et de l'espace. Il essaye de mettre à jour comment, dans ce roman, les structures de l'espace mythique et empirique sont disposées en miroir, en s'appuyant sur les hétérotopies foucaldiennes telles que le miroir, le bateau, le jardin et la bibliothèque. La bibliothèque est le lieu fixe où, en réunissant les documents de la famille Kerbastik, le protagoniste, qui se préoccupe d'écriture, pourra finalement maitriser l'extension du temps dont l'écoulement provoquait en lui une sensation de grande anxiété. Contrairement à l'autobiographie qu'il rédigeait avec difficulté, l'écriture biographique (des membres de sa famille) remet le temps sur les rails en transformant ce dernier d'ennemi en ami. Dans la présente analyse, une place importante revient au mythe celtique de la ville sous-marine d'Ys et à la variante actualisée de l'histoire de Sodome et Gomorrhe, en raison de leur lien étroit avec le raisonnement présidant à la construction du roman.
\end{abstract}

Mots-clefs: structure temporelle et spatiale, Michel Foucault, hétérotopies, mythes celtiques, ville sous-marine d'Ys, autobiographie, le roman artistique

Le temps joue indéniablement un rôle crucial dans le roman artistique, ce genre littéraire typique de la fin du $19^{\text {e }}$ siècle. Il n'est pas question ici de relever une passion particulière pour l'histoire culturelle et ses différentes époques (surtout celle de la Renaissance), mais plutôt de valoriser certains aspects abstraits du concept de temps. Le passage du temps dans la vie de l'homme jette une ombre menaçante sur les artistes qui luttent pour la pérennité, pour conquérir l'éternel. Dans la perspective de la création de valeurs esthétiques, les notions de « temps vide » (« leere Zeit») et de « temps rempli » (« erfüllte Zeit»), proposées par H.-G. Gadamer offrent un tableau suggestif de la différence essentielle qui sépare le temps gaspillé du temps utilisé consciemment et à bonnes fins. (Gadamer 1999 137-153) Un exemple par excellence de cette problématique est donné 
dans le roman de Zoltán Ambrus intitulé « Midas király» (Le roi Midas, 1891). Cependant, c'est à un autre travail, peu connu, que je voudrais consacrer le présent article : «Az utolsó » (Le dernier), roman de Dezső Malonyay en trois tomes publié en 1896. Auteur fécond en son temps, Dezső Malonyay est tombé par la suite dans un oubli presque total. Il faisait partie des écrivains d'orientation française de la deuxième moitié du $19^{\mathrm{e}}$ siècle. Aujourd'hui, il est difficile de croire que Malonyay - tout comme Zsigmond Justh - était considéré comme l'écrivain hongrois le plus connu à Paris dans les années 1890. L'histoire de la littérature hongroise n'a d'ailleurs toujours pas identifié les œuvres qui, concrètement, lui ont valu le respect des lecteurs français. Sans doute n'est-ce pas le maître du roman léger "fin du siècle" qu'ils ont apprécié, bien que l'on doive attribuer clairement ses succès à l'époque en Hongrie á son activité dans ce domaine. Le fait est que si Malonyay s'est vu décerner le prix littéraire de l'Académie francaise, le roman en question n' a connu aucun écho en Hongrie au moment de sa parution.

Le protagoniste du roman $A z$ utolsó est le dernier descendant de l'illustre famille aristocratique des Kerbastik d'Azincourt, il est en outre un authentique bel esprit, mais le sang de ses ancêtres commence à s'user en lui et leur énergie s'amenuise. S'il a des ambitions artistiques, son aspiration manque de persévérance et ne donne pas lieu à une vraie invention. Il apparaît comme une figure étrangère à la vie dont l'existence se déroule dans l'univers des livres. D'ailleurs, il s'identifie volontiers à Don Quichotte. Il cherche lui aussi sa Dulcinée, mais il veille à n'établir de relations trop tendres avec nulle femme. Sur le plan émotionnel, il ne s'attache fortement qu'à sa mère, alors qu'il est terrifié par son père, glacial et peu communicatif. L'expérience la plus traumatisante de sa jeunesse est celle de la mort de son père, dont les circonstances étranges dévoilent la part d'ombre secrète que recèle sa vie recluse : dans ses appartements, le vieux comte célébrait des messes noires, des séances satanistes. Tandis qu'il visite les lieux où les cérémonies étaient organisées, le fils ressent un plaisir mêlé d'horreur. Après les funérailles, Dénes, le serviteur de son père, met le feu à la maison et l'incendie dévore la bibliothèque du jeune comte. Ce dernier voudrait se débarrasser du lourd héritage du passé familial, épouvanté par l'idée que le destin paternel puisse se répéter à travers lui. Il décide alors de déménager à Fousnant, en Bretagne, dans un autre château familial qui est inoccupé et même à l'abandon. Il envisage d'y faire tabula rasa et de recommencer sa vie. Il fait ses valises et se met bientôt en route.

Comme l'a suggéré Foucault dans son essai intitulé «D'autres espaces », le $20^{\mathrm{e}}$ siècle est celui de l'espace. (Foucault 1994 752-762) Autrement dit, dans la vision foucaldienne, le temps s'est arrêté au tournant du siècle pour devenir, en quelque sorte, un système de dispositions spatiales. Dezső Malonyay est un précurseur de cette perception moderne du temps. Dans son œuvre parue aux dernières années du $19^{\mathrm{e}}$ siècle, le héros annonce, dès le commencement de l'his- 
toire, que le temps s'est arrêté : «megállt az idő rám nézve » et que : "mindig egy helyt állott a korom ». (Malonyay 1896 I, 6) Tandis que son train s'ébranle en direction de la lointaine Bretagne, le jeune comte n'a qu'un seul souhait, celui de s'enfermer dans le renoncement et la résignation, dans la plus parfaite monotonie, dans un moment figé pour toujours et de la perspective duquel tout passé semblera infiniment distant et improbable. Il souhaite que les roues cliquettent et roulent au-dessous de lui indéfiniment «sok éjszakán és sok napon át, folyton úgy ». (Malonyay 1896 I, 6) Du reste, le concept de temps statique implique inéluctablement l'impression que le déplacement dans l'espace, lui aussi, est impossible. Le voyageur est saisi d'une illusion d'optique : il a l'impression que le train régresse plus qu'il ne progresse : «nem elöre, hanem ellenkező irányba, visszafelé ». (Malonyay $1986 \mathrm{I}, 6$ ) Plus tard, il prend la voiture de poste et, à chaque relais, il abandonne une partie de ses bagages. Ce geste indique clairement qu'en réalité, c'est dans le temps que le protagoniste voyage en s'efforçant de se débarrasser des morceaux de son passé qui continuent à peser sur lui. Cependant, son avancement virtuel dans l'espace ne semble produire aucun déplacement dans le temps. En route vers Fouesnant, à Rosporden, il est envahi par une force élémentaire qui vient tout droit du passé, car chaque recoin de la maison de la mère Périne respire le souvenir de sa mère que l'on a été hébergée ici lorsqu'elle portait le jeune comte dans son sein. Dès lors, il est bercé par l'oscillation permanente entre le passé et le présent, dans une sorte de rêverie qui éveille en lui une affection étrange pour la fille de ses hôtes, Mathurine. Or il se trouve que Mathurine doit se rendre à Concarneau et les hôtes demandent au jeune comte si leur fille peut l'accompagner. Quand ils arrivent à Concarneau, Mathurine décide d'aller jusqu'à Fouesnant, la destination finale.

De Concarneau, pour atteindre le vieux château des Kerbastik, la route la plus courte passe par la mer, ils continuent donc en bateau. Toujours dans un état d'étourdissement, le jeune comte a de nouveau l'impression d'un retour en arrière - il croit voir une image renversée : « mintha nem is mi mentünk volna, a part suhant elölem távolabb. » (Malonyay 1896 II, 54) Signalons que d'après Foucault, le bateau n'est autre qu'un « morceau flottant d'espace, un lieu sans lieu », quelque chose qui demeure enfermé en soi-même tout en étant abandonné à l'infinité de la mer. (Foucault 1994 762) Symbole ancestral, le bateau voguant dans le lointain élargit la conscience humaine alors que l'aspiration à la haute mer ouvre les écluses au flux de l'imagination artistique. Le comte qui se penche sur la balustrade observe les vagues transparentes et vitreuses. La forêt vert foncé de la végétation du fond marin, les sarments gracieusement entrelacés entre lesquels zigzaguent les bancs de sardines déclenchent son imagination. D'abord, il imagine qu'un homme est couché sous les buissons sous-marins, puis il se représente qu'il s'agit de sa compagne de voyage : «Vagy ha Mathurine feküdnék ott ... Formás idomaira simulna az ázott ruha, vörös haja szétterülve lebegne feje körül, 
belepné az arcát, a mellét, az indák köré fonódnék, apró, gyöngyházas kagylók telepednének a vállaira, s úgy ereszkedném le az ölébe, mint az a medusa ... » (Malonyay 1896 II, 58) Le comte se remet à peine de la torpeur étrange de sa vision quand Mathurine commence à lui raconter qu'ici où la mer éblouissante : « olyan szép sötétkék szinü és összeölelkezik az éjszakára barnuló éggel, - egy nagy város volt valaha. » (Malonyay 1896 II, 58) Ses paroles font revivre l'histoire d'Ys, l'ancienne ville engloutie par la mer. Selon les ouï-dire qui circulent dans la région, de temps en temps, les vieilles femmes de pêcheurs voient à travers le miroir d'eau les toits brillants d'Ys, la cavalcade panachée de la foire du marché, elles entendent distinctement le carillon des églises et tout est «csupa, csupa kastély, meg torony, meg ezer meg ezer nyitott ablak! (Malonyay 1896 II, 60) Quand Mathurine termine son récit, le bateau arrive au port et les figures spatiales mythiques se matérialisent dans l'espace réel de Fouesnant : «Ott a kastély'Mintha az is a meséböl lett volna, a sáncokként huzódó fövénypadok mögött, a fekete partsziklák fölött látszott a kastély. » (Malonyay 1896 II, 60)

Comme 1'observe Ernst Cassirer, les espaces mythiques ne constituent pas des lieux au sens empirique-physique : ils sont dominés par des traits magiques et tout ce qui se passe là-bas est opéré par des dichotomies telles que le salut et la perdition, l'accessibilité et l'interdiction, la bénédiction et la malédiction, l'ambiance familiale et l'étrangeté. (Cassirer 1985 95-111) Cependant, la manière dont la conscience esthétisante relie les correspondances cachées entre les diverses figures spatiales du roman de Malonyay est très particulière. Tout comme le narrateur, à la première personne, perçoit dans l'espace de sa vie quotidienne, jour après jour, que pour lui « le temps s'est arrêté », ainsi cela devient-il l'expérience existentielle des habitants d'Ys, engloutie par la mer :

\footnotetext{
« Mióta a várost elnyelték a hullámok, minden, minden ugy maradt, ahogyan akkor

volt és azóta mindenki azt csinálja, amit akkor csinált. A rokka mellett ülö asszonyok

azóta folyton pörgetik az orsót, a posztókereskedök azóta folyton ugyanazt a posztót

mérik, ugyanazoknak a vevöknek. És ez így lesz, így, míg csak meg nem váltja öket a

jó szerencse, amikor a város ujra elötünik majd a habok alul.»
}

(Malonyay 1896 II, 60)

Les détails de l'action qui se déroulent respectivement dans l'espace empiriquephysique et dans l'espace mythique se superposent en formant une sorte d'image miroir. Le principe de la symétrie en miroir est utilisé de façon consciente ; ce mécanisme apparaît aussi dans le fait que, d'un côté, le père sataniste du jeune comte célébrait des messes noires au fond de ses chambres secrètes, de l'autre, un évêque dans chacune des cent églises de la ville d'Ys murmure la liturgie de la 
sainte messe. L'apparition d'un des pêcheurs de Douarnenez ne fait que renforcer le parallèle. Ce dernier raconte qu'un jour, lors d'une plongée sous-marine, il a vu un prêtre préparant la messe dans une des églises d'Ys dire à un enfant que ce devait être à lui de servir. Offrant une interprétation à son récit, le curé local dit au pêcheur qu'il aurait dû lui-même accepter de servir la messe à la place de l'enfant, car il aurait ainsi sauvé la ville d'Ys de la malédiction. Dénes, le serviteur du vieux comte Kerbastik ne dispose pas, quant à lui, d'un tel choix. Au lieu d'une cloche, c'est toujours l'inexorable sonnette qui le convoque à l'endroit du « service ». Il sait très bien ce qu'il devra faire, mais il n'aura jamais la force d'éliminer son maître, d'exécuter ainsi l'acte de violence capable de sauver tout le monde. C'est le même message qui se dégage de l'histoire de la femme goémonière qui trébuche sur la plage : «... mikor fölkelt, egy rengeteg kaput látott maga elött és az a rengeteg kapu szépen föltárult és ö bement rajta és egy pompás városba érkezett. » (Malonyay 1896 II, 61) Selon la logique du conte, l'acte rédempteur n'a pas lieu parce que, faute d'argent, la femme ne peut rien acheter parmi les splendides étalages. Or la malédiction affligeant Ys ne peut être levée que de cette manière. Ici, ce n'est pas seulement la présence de la « multitude de portes » qui suggère une analogie directe - le site des rites sataniques étant également accessible à travers une multitude de portes cachées -, mais aussi la soie des nombreux vêtements coûteux accumulés devant la porte des magasins de la ville sous-marine ( sok drága köntösre való selyem »). Ces éléments visuels, eux aussi, évoquent les événements ayant eu lieu à la résidence du vieux comte. Dans ce lieu particulier, quand Dénes arrache la dernière porte d'armoire, le jeune Kerbastik voit devant lui les mêmes vêtements luxueux : " nöi köntösök vannak benne. " (Malonyay 1896 I, 135) Mais l'élément capital qui réunit les deux univers spatiaux est la clef. Ce motif émerge dès le commencement du roman, puis il demeure à travers le trousseau de clefs en possession de Dénes, qui donne accès aux chambres secrètes du vieux comte, pour enfin s'accomplir avec les clefs du château de Fouesnant que la mère du jeune Kerbastik a déposées, il y a fort longtemps, chez le curé local. Se joint à ce fil de signification la clef en argent du mythe celtique ouvrant la porte d'écluse de la fée Ahès-Dahut, dont l'acquisition par des mains non autorisées scellera pour toujours le destin de la ville tombée dans le bourbier du vice. Évidemment, la légende d'Ys n'est autre que la répétition de l'histoire biblique de Sodome et Gomorrhe. De fait, l'univers transcendantal celtique-breton et celui du christianisme ne cessent de se mélanger dans le cours des événements qui s'entremêlent, à leur tour, dans l'espace mythique et empirique-physique. À ce propos, il suffit peut-être de s'en remettre aux affinités respectives de Gradlon et d'Ahès-Dahut ou à l'effet sur les consciences des superstitions païenne des pêcheurs, bien connues de l'abbé Conan. Toutefois, chacun des deux schémas mythiques implique des éléments qui peuvent être mis en relation directe avec les questions existentielles qui tourmentent le héros 
principal. Parmi ces éléments figure le dénouement des bacchanales d'Ahès-Dahut, aboutissant inexorablement au meurtre et en cela correspondant essentiellement aux rites sacrificiels de la messe noire. Apparemment, derrière la surface visible du miroir d'eau qui moutonne paisiblement, des mondes invisibles peuvent s'ouvrir - de la même façon qu'une personnalité qui, de l'extérieur, semble dure comme la pierre peut se désintégrer de manière quasiment inaperçue sous la force érosive des instincts libérés. Le seul antidote à cette menace génétiquement déterminée - d'après le médecin de famille du comte - est le travail artistique créateur. Le dernier Kerbastik d'Azincourt s'y prépare justement. Son plus grand désir est de transposer l'expérience de l'espace mythique dans l'espace esthétique afin qu'en apprivoisant les démons du monde magique, il puisse élaborer divers moyens de rendre compte des expériences vécues. Cependant, s'il veut réussir dans son entreprise, il aura besoin d'un assistant qui se sente à l'aise dans la réalité quotidienne aussi bien que dans l'univers du mythe servant de matière première esthétique. Le souvenir du fabuleux voyage en mer et le chuchotement des ombres respirant la brume des légendes celtiques dirigent ensemble les pensées du jeune comte vers Mathurine.

De fait, Dezső Malonyay révèle dès les premières pages que le lecteur va lire une autobiographie. Le dernier comte Kerbastik fait le projet d'enrouler le fil du temps par l'acte d'écriture afin de s'élever à un niveau supérieur de la connaissance de soi. Dans le même temps, le paradoxe de l'autobiographie tient précisément au fait que l'effort entrepris en vue de restaurer la fugacité « by autography (the prosopopeia of the voice and the name) deprives and disfigures to the precise extent that it restores » (Paul de Man 1984 81) ; ainsi, au lieu de mettre en évidence une connaissance de soi fiable, elle en démontre au contraire l'impossibilité. Paul de Man signale que le profil autobiographique s'inscrit dans une structure spéculaire particulière ("specular structure ») au sein de laquelle le narrateur et le matériau de la narration se définissent dans une sorte de substitution réciproque et réflexive. Selon son argumentation, le détail autobiographique prend forme au cours d'un processus d'alignement réciproque de l'auteur et de son matériaux, donnant lieu à une structure qui nait à la fois de la distinction et de la similarité : "This specular structure is interorized in a text in which the author declares himself the subject of his own understanding. " (Paul de Man 1984 70) Le héros de Malonyay fait face à cette situation, sans parvenir à la surmonter. Plongé dans l'ardeur du travail, il se heurte en permanence au problème de son incapacité à écrire : "Ha végignézek egy teleirt lapot s analizálom rajta a törléseket, javitásokat, megkapom rajta az én egész életem pszikologiáját. Nyilvánvaló : - most, hogy igy követem s ugyszólva ujra átélem önmagamat, ez annyira enervál, hogy szinte másodszor okoskodom bele magamat a tehetetlenségbe. 》 (Malonyay 1896 II, 145-146) 
Pour sortir de son état apathique, il faudrait évidemment qu'il puisse bénéficier d'une inspiration extérieure. Cependant, au cours du jeu pendulaire et frénétique de rapprochement et d'éloignement par rapport à Mathurine, le dilemme semble au contraire ne pas cesser de s'intensifier. Face au jeune comte esthétisant, la jeune et robuste bretonne, débordant de vitalité, apparaît tantôt dans l'optique du mythe et de son mystère plein d'élévation, tantôt dans la banalité presque gênante de son impulsivité crument sexuelle. À travers la figure de Mathurine, en tant que « conteuse », apparaît parfois la silhouette d'Ahès-Dahut, la séductrice que l'univers des croyances celtiques a associée avec l'abondance, la fertilité et la richesse aussi souvent qu'avec la fornication effrénée ou la perdition. Un jour, Mathurine offre un coing au jeune comte. Dans la mythologie celtique, le coing est le symbole de la fertilité. Selon certains spécialistes de l'herméneutique biblique, la pomme défendue du Jardin d'Éden aurait été un coing à l'origine. Pourtant, on n'apprend pas grand-chose sur le mode de vie de la jeune femme. Sans doute aurait-elle peu de scrupules moraux à tromper Grégoire, son mari boiteux. Mais il n'apparaît pas clairement dans la narration qu'elle ait une véritable liaison avec le serviteur du comte, ou si ce n'est qu'une fantaisie apparaissant sur l'horizon mental de Kerbastik, toujours à la recherche d'analogies. Son serviteur à Fouesnant s'appelle Dénes, tout comme son serviteur actuel, et nous savons déjà que le vieux comte avait effectivement pris la maîtresse de son valet. Et le jeune comte de constater avec résignation : "Ime, az elsö asszony, akit a sors elém taszit, hozza magával azt, amit el akartam veszteni, az apám emlékét ... És milyen megdöbbentöen hasonlit ez az eset az övéhez ! » (Malonyay 1896 II, 149)

Dans son œuvre intitulée "Les mots et les choses », Michel Foucault rappelle que l'histoire de Don Quichotte n'est autre que celle de la quête de ressemblances. Le chevalier se retrouve au bord de la folie parce qu'il parvient à intégrer partout les analogies trouvées dans les indices qu'il remarque. Ici, Foucault souligne que dans l'histoire culturelle de l'humanité, c'est précisément pendant la Renaissance que la relation entre le signifiant et le signifié s'est rompue. Depuis lors, les mots ne signifient plus les choses et l'écriture n'est plus la prose du monde. (Foucault 1966 60-64) Peinant sur son autobiographie, le comte Kerbastik aura la même expérience quand il se plaint que : «nem találok kello" szavakat a kifejezésre » ou « hideg, üres valamennyi, amelyet én találok. » (Malonyay 1896 II, 145) Si cette attitude de Don Quichotte laisse constamment des impressions négatives sur ses efforts artistiques stériles, elle fait aussi échouer ses timides tentatives amoureuses. Puisque toute sa vie ne fait qu'imiter le livre et l'écriture, il ne sait que faire dans la réalité d'une femme de chair et de sang. Lorsque finalement, Mathurine lui rend visite au château, rien ne se passe entre eux. Comme la femme déçue finit par le constater : "No ezért ugyan kár volt idebolonditani az embert. » (Malonyay 1896 III, 18) 
L'(auto)biographie est une création intellectuelle spéciale. Virginia Woolf, par exemple, l'a plus considérée comme de l'“artisanat" que de l'art. C'est un genre qui navigue essentiellement entre les possibilités de l'art et celles de la science : pour l'articulation linguistique des expériences vécues, il faut absolument une imagination qui le porte plutôt vers l'art, mais le respect des faits le fait pencher quand même vers la philologie, c'est-à-dire vers la science. (Woolf 2015) L'(auto)biographie va devenir un point de bifurcation particulier des efforts du jeune comte. Pendant qu'il écrit l'histoire de sa vie, il s'oriente d'abord vers les genres littéraires au sens classique. Sous le charme des contes de Mathurine, il cherche un sujet qui lui permette d'aller et venir librement « a mese és a valóság szivárványhídján », mais, pendant quelque temps, il espère aussi trouver de la matière pour « valami szép, gyengéd regényre ». (Malonyay 1896 II, 109, 149) Plus tard, son intérêt se tourne vers la pièce de théâtre. Sa forte faculté d'abstraction se manifeste dans le drame qu'il se propose d'écrire et qui mettra en scéne non des gens véritables mais seules les ombres projetées des personnages. Pourtant, ses trouvailles ne restent que des copeaux d'idées, des ambiances passagères, des impressions fugitives desquels rien ne se matérialise, parce que le comte manque d'invention, de faculté d'imagination créatrice. Un symptôme réitéré en est le vertige quelquefois presque gênant qu'il éprouve en haute mer, dans l'empire de l'imagination productive. En même temps, il s'avère aussi que l'écriture d'une (auto)biographie ne sera pas une arme efficace contre le pouvoir du temps qui subjugue tout. En effet, il est incapable d'éliminer la circulation perpétuelle. Pas même au niveau de l'expérience : «Hisz ime, egyenesen megyek vissza oda, ahonnan menekültem, shogy annál evidensebb legyen, még le is irom a naponkint tett apró utat. » (Malonyay 1896 II, 119) Dès lors, il ne lui reste que l'autre direction : l'historiographie et l'univers de la science.

Outre le jardin représentant l'équilibre, la paix et le bonheur, l'endroit le plus important dans la structure spatiale du roman est sans doute la bibliothèque. D'ailleurs, Foucault classe le musée et la bibliothèque parmi les hétérotopies les plus typiques de la culture occidentale du $19^{\mathrm{e}}$ siècle. C'est la réaction défensive par excellence de l'homme moderne par laquelle il essaie de repousser les attaques perpétuelles du temps. La création de bibliothèques et de musées toujours plus nombreux est évidemment motivée par l'intention d'enfermer chaque époque et chaque goût dans un lieu concrètement défini. L'on ne peut se débarrasser de la diffusion floue et angoissante du temps que par la localisation, et cela en assurant l'accumulation infinie du temps dans un seul et même espace immobile : «le projet d'organiser ainsi une sorte d'accumulation perpétuelle et indéfinie du temps dans une lieu qui ne bougerait pas. » (Foucault 1994 759) C'est par cette stratégie que le héros de Dezső Malonyay tente maintenant de maîtriser le temps. Il se retire dans la tour d'ivoire de la science où il décide d'étudier avec une attention obstinée les livres consacrés à l'histoire de sa famille qu'il a trouvés 
dans l'observatoire du château. Mais il ne se contente pas de cela. Il ordonne à son intendant de se rendre aux archives nationales afin de se procurer une copie de tous les documents sur lequel figure le nom de Kerbastik. Des établissement de toute la France se voient peu à peu inclure dans la portée de la recherche et un nombre croissant de livres liés au sujet arrivent au château. Le comte réalise ainsi, mot à mot, le programme foucaldien quand - au lieu de voyager à travers le pays il observe avec satisfaction et d'un point de repère statique l'afflux du temps dans sa bibliothèque créée ad hoc. Le but auquel il a échoué pendant ses voyages, en se déplaçant constamment dans le temps, il semble désormais y parvenir enfin : il fait un pied de nez au temps. Auparavant, il a eu beau chaque jour abandonner volontairement un pièce de ses bagages, il était incapable de se débarrasser du poids de son passé hérité. Mais, maintenant, c'est le temps maîtrisé qui vient à lui, un temps qu'il peut désormais mettre à son service et qui d'ennemi, devient son ami dans la fièvre du travail. Sans s'interrompre, il dépouille la matière reçue. Le fruit de ses efforts tenaces sera un travail historique de six tomes qui lui vaudra le titre d'académicien. Contrairement à l'autobiographie rédigée dans la douleur, il découvre finalement la joie de la «création »-joie qu'il n'a jamais éprouvée au cours de ses précédentes tentatives littéraires - grâce à la biographie et tout porte à croire qu'il réussit, par son geste créateur, à remettre le temps sur les rails.

\section{Références}

Cassirer, Ernst, 1985. Mythischer, ästetischer und theoretischer Raum. In: Orth, Ernst Wolfgang, Krois, John Michael, ed. Symbol, Technik, Sprache. Aufsätze aus den Jahren 1927-1933. Hamburg: Felix Meiner Verlag, 93-111.

De Man, Paul, 1984. Autobiography as De-Facement. In: The Rhetoric of Romanticism. New York: Columbia Press, 67-81.

Foucault, Michel,1994. Des espaces d'autres. In: Defert, Daniel, Ewald, François and Lagrange, Jacques, ed. Dits et écrits 1954-1988, IV, 1980-1988. Paris: Gallimard, 752-762.

Foucault, Michel, 1966. Les choses et mots. Paris: Gallimard, 60-64.

Gadamer, Hans-Georg, 1999. Über leere und erfüllte Zeit. In: Gesammelte Werke, IV, Tübingen: Mohr Siebeck.

Malonyay, Dezső, 1896. Az utolsó I-III. Budapest: Athenaeum.

Woolf, Virginia, 2015. The art of biography. In: The Death of the Moth, and other Essays. Adelaide: eBook. 
